Review Article

\title{
New Insights into the Role of Mitochondrial Dynamics and Autophagy during Oxidative Stress and Aging in the Heart
}

\author{
Yoshiyuki Ikeda, ${ }^{1}$ Sebastiano Sciarretta, ${ }^{1,2}$ Narayani Nagarajan, ${ }^{1}$ Speranza Rubattu, ${ }^{2,3}$ \\ Massimo Volpe, ${ }^{2,3}$ Giacomo Frati, $^{2,4}$ and Junichi Sadoshima ${ }^{1}$ \\ ${ }^{1}$ Cardiovascular Research Institute, Department of Cell Biology and Molecular Medicine, Rutgers New Jersey Medical School, \\ 185 South Orange Avenue, Medical Science Building G-609, Newark, NJ 07103, USA \\ ${ }^{2}$ IRCCS Neuromed, Via Atinense 18, 86077 Pozzilli, Italy \\ ${ }^{3}$ Division of Cardiology, Department of Clinical and Molecular Medicine, Faculty of Medicine and Psychology, Sapienza University, \\ Via di Grottarossa 1035-1039, 00189 Rome, Italy \\ ${ }^{4}$ Department of Medico-Surgical Sciences and Biotechnologies, Sapienza University, Corso della Repubblica 79, 04100 Latina, Italy
}

Correspondence should be addressed to Sebastiano Sciarretta; sebastianosciarretta@yahoo.it and Junichi Sadoshima; sadoshju@njms.rutgers.edu

Received 28 March 2014; Revised 5 June 2014; Accepted 18 June 2014; Published 15 July 2014

Academic Editor: Neelam Khaper

Copyright (C) 2014 Yoshiyuki Ikeda et al. This is an open access article distributed under the Creative Commons Attribution License, which permits unrestricted use, distribution, and reproduction in any medium, provided the original work is properly cited.

\begin{abstract}
The heart is highly sensitive to the aging process. In the elderly, the heart tends to become hypertrophic and fibrotic. Stiffness increases with ensuing systolic and diastolic dysfunction. Aging also affects the cardiac response to stress. At the molecular level, the aging process is associated with accumulation of damaged proteins and organelles, partially due to defects in protein quality control systems. The accumulation of dysfunctional and abnormal mitochondria is an important pathophysiological feature of the aging process, which is associated with excessive production of reactive oxygen species. Mitochondrial fusion and fission and mitochondrial autophagy are crucial mechanisms for maintaining mitochondrial function and preserving energy production. In particular, mitochondrial fission allows for selective segregation of damaged mitochondria, which are afterward eliminated by autophagy. Unfortunately, recent evidence indicates that mitochondrial dynamics and autophagy are progressively impaired over time, contributing to the aging process. This suggests that restoration of these mechanisms could delay organ senescence and prevent age-associated cardiac diseases. Here, we discuss the current understanding of the close relationship between mitochondrial dynamics, mitophagy, oxidative stress, and aging, with a particular focus on the heart.
\end{abstract}

\section{Introduction}

Over the past few decades, the human life span has been extended and is expected to increase further in the next few years with a supplementary prolongation of life expectancy. Consequently, the prevalence of age-related diseases, such as cardiovascular diseases (CVD), is increasing [1]. Hypertension, dyslipidemia, diabetes mellitus, and metabolic syndrome increase with age and play a key role in the development of CVD, which is a major cause of chronic disability, morbidity, and mortality in the elderly $[2,3]$. The aging process is associated with accumulation of damaged proteins and organelles that is partly attributable to a decline in the maintenance of protein quality control systems [46]. In addition, a critical pathological feature of the aging process is the development of mitochondrial abnormalities. Reactive oxygen species (ROS) progressively accumulate during aging as a result of impairment of mitochondrial oxidative phosphorylation, misfolded protein accumulation, and an imbalance between the expression of oxidant and antioxidant proteins [7-11]. ROS accumulation, in turn, further attenuates the bioenergetic function of mitochondria by causing mutations in mitochondrial DNA (mtDNA), impairing the tricarboxylic acid cycle (TCA) and the electron transport chain complexes. This causes a vicious cycle that further and progressively aggravates oxidative stress in 
tissues and mitochondrial dysfunction in aged organs [911]. Therefore, an optimally functioning regulation system that is able to eliminate damaged cellular constituents and organelles, in particular mitochondria, is critical to maintain cellular homeostasis during aging. As recently highlighted, cardiomyocytes are not terminally differentiated cells and have the capacity to reenter the cell cycle, but this process seems to be quenched by a limited turnover, making them reliant on these mechanisms to remove damaged or longlived cellular components or proteins $[12,13]$.

Mitochondrial dynamics, particularly mitochondrial fusion and fission, are important processes for mitochondrial homeostasis $[14,15]$. In particular, mitochondrial fission allows for selective elimination of damaged mitochondria by autophagy $[16,17]$. Autophagy, which is derived from the Greek words "autòs" ( $\alpha \dot{v} \tau o ́ s$, for self) and "phagèin" ( $\varphi \alpha \alpha \omega$, for eating), is an evolutionarily conserved system that targets damaged or long-lived proteins and organelles for degradation through lysosomes [16, 18]. Autophagy can be either nonselective or cargo-specific. Mitochondrial-specific autophagy is referred to as mitophagy. Accumulating lines of evidence indicate that autophagy and mitophagy play a crucial role in the regulation of cardiac homeostasis and response to stress [17-19].

It is now known that abnormalities in mitochondrial dynamics and autophagy contribute to the aging process [1416]. This review will deal with the current understanding concerning the close relationship between mitochondrial dynamics, mitophagy, oxidative stress, and aging in the heart.

\section{Oxidative Stress, Mitochondria, and Aging}

Oxidative stress is reported to increase in hypertension, hyperlipidemia, diabetes mellitus, and smoking, where it contributes to the development of atherosclerosis [20]. Oxidative stress is also known to be involved in the development of cancer and Alzheimer's disease associated with aging [21, 22]. ROS are constantly generated in cells as a byproduct of oxygen metabolism [9] since they are important cell signaling molecules at physiological levels [23]. For instance, we recently demonstrated that ROS production by Nox4, a member of the NADPH oxidase family, in the endoplasmic reticulum is important for autophagy induction and cell survival during cardiomyocyte energy deprivation [24]. However, excessive ROS induce an imbalance between the oxidant and antioxidant responses in cells [9].

Oxidative stress is increased during the organ aging process $[9,11,25]$. Production of ROS is progressively enhanced over the years in different organelles. We have demonstrated that mitochondrial expression of Nox4 is upregulated in the senescent heart, contributing to ROS production and development of cardiac abnormalities [26]. In fact, mice with cardiac-specific overexpression of Nox4 develop cardiomyopathy in the late phase of life [26]. Recent evidence from lower organisms also indicates that the homolog of Nox4 in yeast is upregulated in the endoplasmic reticulum in response to mitochondrial dysfunction [27], a mechanism that would contribute to the aging process. P66 adaptor protein also accumulates progressively in aged mitochondria, and p66 deletion significantly extends life span in mammals [28]. ROS favor accumulation of misfolded proteins that, in turn, further enhance oxidative stress [29]. Of note, ROS not only damage proteins by directly oxidizing them but also have been shown to impair the activity of immunoglobulin heavy chain binding protein (Bip) and protein disulfide isomerase (PDI) in the endoplasmic reticulum of the mouse liver, thereby affecting the protein folding process during aging [30].

Antioxidant gene expression and activity are also affected during aging. The capacity for activating manganese superoxide dismutase (MnSOD) and catalase expression during oxidative stress and exercise is reduced over time in skeletal muscle [31]. In addition, reduction of sirtuins (SIRT) promotes ROS production during aging. SIRT3 deacetylates and activates MnSOD in the mouse liver [32]. SIRT3 also increases the activity of isocitrate dehydrogenase 2 during aging, thereby stimulating the tricarboxylic acid (TCA) cycle in the ear system, liver, and brain in mouse [33]. This event increases the amount of mitochondrial NADPH and protects against oxidative-stress-induced damage by increasing the ratio of reduced-to-oxidized glutathione. SIRT1 also promotes antioxidant defense through the activation of Forkhead box protein $\mathrm{O} 1$ (FOXO1) signaling in multiple mammalian cell lines [34]. The SIRT3 expression level was found to be reduced in aged skeletal muscle [35]. Although SIRT1 expression seems to increase in the elderly [36], ROS were found to be able to promote SIRT1 degradation through its oxidation in lung epithelial cells [37]. Furthermore, SIRT1 activity is reduced during aging due to a dramatic reduction of NAD+ levels in mouse skeletal muscle [38]. Of note, ROSdependent AMP-activated protein kinase (AMPK) inhibition could also potentially lead to a reduction of antioxidant defenses during aging. In fact, AMPK can stimulate the antioxidant response through FOXO1 activation [39]. We recently found that ROS can directly oxidize AMPK at cysteines 130 and 174 and inhibit its activity in cardiomyocytes [40].

In apparent contrast with this evidence, it has been shown that impaired insulin/insulin growth factor 1 (IGF-1) signaling and deletion of superoxide dismutase 2 extend life span in $C$. elegans through a mild increase in oxidative stress. This low accumulation of ROS is not sufficient to promote cellular damage, whereas it is able to trigger several cellular adaptive responses that are able to increase the organism's resistance to later stronger stresses $[41,42]$. Therefore, despite the fact that pathological increases in ROS are maladaptive and reduce longevity, transient and physiological ROS activate adaptive signaling pathways that can promote survival.

Mitochondrial dysfunction represents a common feature of the aging process [43]. ROS progressively promote mtDNA mutations and deletions that accumulate over time, leading to progressive reduction of the number of mtDNA copies $[44,45]$. Oxidative damage of mtDNA contributes to impairment of the electron transport chain, to mitochondrial uncoupling, and, ultimately, to bioenergetic dysfunction of mitochondria characterized by reduced ATP production and further accumulation of ROS [46, 47]. In support of the 
importance of mtDNA mutations in the aging process, it has been observed that a mouse model with defective mtDNA polymerase (PolgA) displays a high rate of mtDNA mutations and a reduced life span, which is associated with early signs of senescence such as osteoporosis, hair and weight loss, anemia, and cardiac hypertrophy [48]. In addition to damaging mtDNA, ROS can directly impair the TCA cycle, particularly by targeting aconitase [49]. ROS can also impair the enzymes involved in oxidative phosphorylation, particularly the enzymes containing an iron-sulphur cluster, in eukaryotic cells [50]. Finally, oxidative stress is associated with mitochondrial transition pore opening, which promotes necrosis and cell death in mammalian cells [51]. The observation that systemic mitochondrial overexpression of catalase extends life span and delays the aging process in mice is evidence that mitochondrial ROS strongly contribute to the aging process [52]. In addition, mitochondrial catalase prevents age-associated mitochondrial dysfunction and insulin resistance [53]. Of note, overexpression of catalase in subcellular organelles other than mitochondria failed to extend life span in mice, and overexpression of different isoforms of superoxide dismutase and peroxidase enzymes was not associated with a significant extension of longevity $[54,55]$. These results suggest that accumulation of ROS, more specifically hydrogen peroxide, in mitochondria, but not in other organelles, contributes to the aging process in mammals. This is likely due to a progressive reduction of mitochondrial function $[54,55]$. It should be pointed out that a reduction of sirtuin activity during aging can also contribute to mitochondrial dysfunction independently of ROS. For example, SIRT3 can stimulate oxidative phosphorylation by directly activating the electron transport chain complexes in the heart, kidney, and liver [56]. SIRT3 deletion can also accelerate age-dependent cardiac hypertrophy by the lack of deacetylation of cyclophilin D at lysine 166 [57]. This mechanism prevents age-dependent mitochondrial transition pore opening and ROS accumulation.

Mitochondrial abnormalities and dysfunction play a fundamental pathologic role during the aging process in the heart $[58,59]$. This is not surprising based on the evidence that the heart is an organ that relies on extensive energy production to support the cardiac cycle. Enlarged mitochondria with membrane and matrix abnormalities accumulate in the aged heart $[58,59]$. Mitochondrial ROS production and mtDNA oxidative damage also progressively accumulate $[58,59]$. As a result, increased mitochondrial ROS can promote cardiac hypertrophy and cardiac fibrosis $[58,59]$. We previously showed that aging upregulates Nox4 [26] and that upregulation of Nox4 in the nucleus promotes cardiac hypertrophy through oxidation and nuclear export of histone deacetylase 4 (HDAC4) [60]. ROS accumulation also promotes cardiac inflammation, with increased recruitment of inflammatory cells, fibroblast activation, and, ultimately, hypertrophy and fibrosis [61]. Diastolic dysfunction is highly prevalent in the aged heart $[58,59]$ due to increased fibrosis as well as direct oxidation and inhibition of sarcoplasmic reticulum $\mathrm{Ca} 2+$ ATPase (SERCA2a) [62] and reduction of mitochondrial function [63]. Systolic dysfunction may also occur during aging, through mitochondrial-dysfunction-dependent apoptosis and impairment of energy production $[58,59,63,64]$.

Therefore, it appears clear that the mechanisms that guarantee quality control in mitochondria may play a fundamental role during the aging process by limiting the accumulation of damaged mitochondria and organ damage. Recently, it has been reported that mitochondrial fusion and fission represent important mechanisms for preserving mitochondrial function [14]. In fact, fusion improves mitochondrial oxidative capacity and limits age-dependent mtDNA mutations, whereas fission allows the maintenance of functional mitochondria by segregating damaged parts. Importantly, damaged mitochondria undergo quality control through selective elimination by mitophagy [17].

\section{Autophagy and Cardiac Aging}

Autophagy plays a crucial role in the degradation of long-lived proteins and organelles. These properties make autophagy a crucial mechanism for maintaining tissue homeostasis during the aging process [16, 18]. Three types of autophagy have been identified: microautophagy, chaperone-mediated autophagy, and macroautophagy (commonly referred to as autophagy). Microautophagy is characterized by the direct delivery of sequestered cellular constituents into lysosomes. In microautophagy, the lysosomal membrane invaginates cytosolic components [16, 18]. In chaperone-mediated autophagy, chaperones such as heat-shock proteins help deliver macromolecules to the lysosomes. In macroautophagy, hereafter referred to as autophagy, a small vesicular sac, called the isolation membrane or phagophore, is initially formed. The phagophores enclose cytosolic long-lived proteins and organelles, resulting in the formation of double-membraned structures called autophagosomes. The autophagosomes then fuse with lysosomes, which leads to the degradation of the sequestered cellular constituents through digestion of the cargo by lysosomal hydrolases [16, 18].

The autophagic process consists of the following stages: initiation/nucleation, elongation, and maturation/retrieval of autophagosomes (Figure 1) [16-18, 65]. These processes require autophagy-related gene (Atg) proteins that are evolutionarily conserved and were initially identified in yeast [66].

Autophagy is deeply involved in the regulation of cardiac homeostasis and response to stress [19, 67-69]. Energy deprivation is a strong stimulus for autophagy. In response to nutrient deprivation, hypoxia and ischemia, autophagy is upregulated to digest damaged proteins and organelles, a process that also recycles amino acids for energy production, new enzyme synthesis, and, ultimately, maintenance of cellular functions [19, 67-69].

Autophagy is a critical mechanism for the aging process. Inhibition of autophagic proteins, such as Atg1, Atg7, Atg18, and Beclin1, significantly shortens life span in lower organisms [70]. In mammals, deletion of autophagic proteins in organs, such as the liver $[71,72]$, brain $[73,74]$, pancreas [75], and heart [76], is associated with early signs of senescence and dysfunction characterized by intracellular misfolded protein accumulation and the presence of aberrant mitochondria. On 


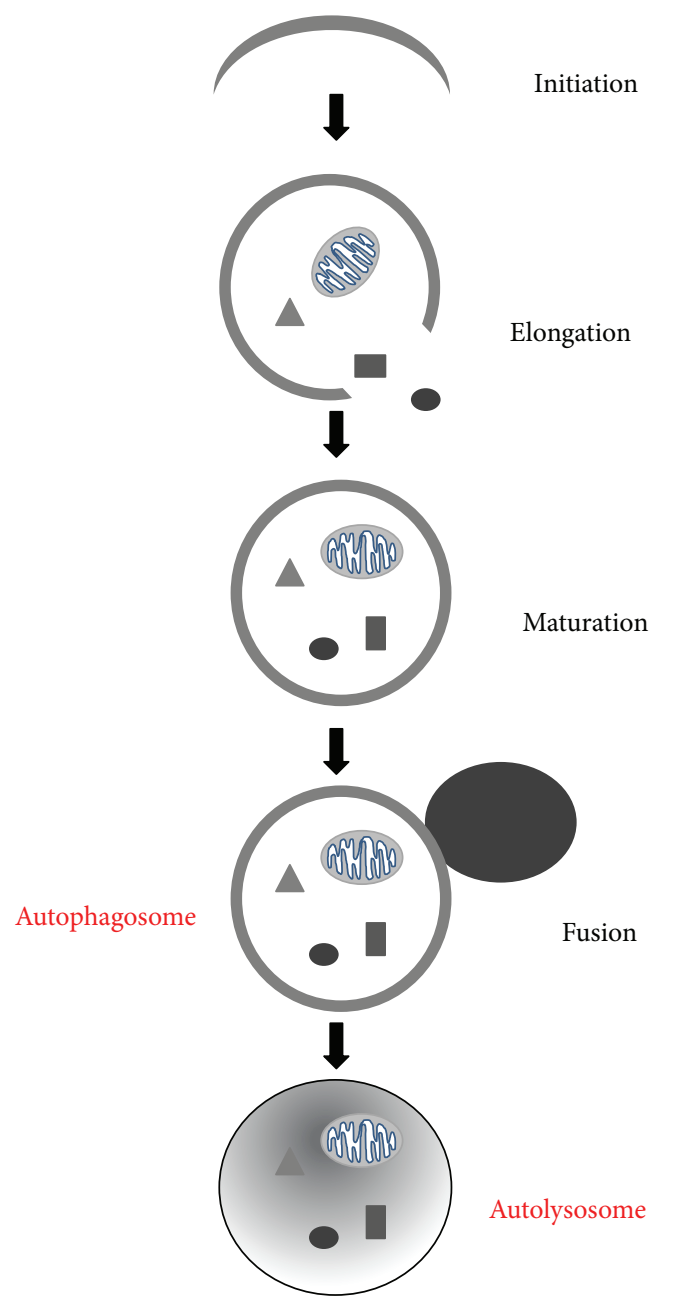

FIGURE 1: Autophagy machinery. Schema representing the four stages of the autophagic process.

the other hand, whether autophagy activation is sufficient to extend life span in mammals is still under investigation. Inhibition of mTORC1 [77] and caloric restriction [78], two conditions that have been shown to enhance longevity, strongly promote autophagy [16]. In Drosophila, the protective effect of rapamycin on aging is abolished by concomitant inhibition of autophagy by Atg 5 knockdown [79]. Interestingly, autophagy declines with age, and the impairment of autophagy contributes to neurodegeneration, insulin resistance, cardiac hypertrophy and dysfunction, and osteoarthritis [16]. Autophagy inhibition further worsens mitochondrial abnormalities and dysfunction and aggravates the accumulation of misfolded proteins [16]. This evidence suggests that autophagy inhibition during aging may play a significant causative role in age-associated diseases.

Accumulation of ROS and mitochondrial dysfunction appears to contribute to autophagy inhibition during aging [16]. In fact, ROS promote accumulation of oxidized proteins that tend to aggregate together in lysosomes. This accumulation of misfolded proteins further promotes ROS production, causing membrane lipid peroxidation and aggravating protein aggregation. When mitochondria are sequestered by lysosomes or are severely damaged and release their contents, mitochondrial peroxides accumulate in lysosomes and create a Fenton reaction that promotes crosslinking reactions among the accumulated misfolded proteins, resulting in formation of the pigment lipofuscin [80]. Lipofuscin accumulates in lysosomes and inhibits lysosomal activity [81]. As a result, autophagic flux is likely inhibited. The lysosome-associated membrane protein $2 \mathrm{a}$ (LAMP2a) is also downregulated during the aging process, contributing to the impairment of autophagic flux [82]. Of note, reactivation of LAMP2a restores both chaperone-mediated and conventional autophagy [82]. Also of note, multiple genes involved in autophagosome formation are reduced during aging, suggesting that the autophagy process is impaired at multiple levels [16].

Autophagy is inhibited in the aged heart [76]. It has been shown that mice with cardiac-specific deletion of Atg5 protein, through constitutive $\alpha M H C-C R E$ expression, develop a dilated cardiomyopathy during aging, characterized by severe systolic dysfunction, sarcomeric disarray, and accumulation of dysfunctional and abnormal mitochondria [76]. This evidence indicates that autophagy is required for cardiac homeostasis during aging, and autophagy inhibition contributes to cardiac senescence. Rapamycin and caloric restriction, both of which can reactivate autophagy, were shown to reverse age-dependent cardiac hypertrophy and diastolic dysfunction [83-86].

\section{Mitochondrial Elimination by Autophagy}

Mitochondrial quality control is essential for maintaining tissue homeostasis $[14,15]$. Mitochondrial quality control is regulated by the balance between the elimination of mitochondria through autophagy and mitochondrial biogenesis $[14,15]$. Mitochondria are dynamic organelles that are constantly undergoing fission and fusion to adapt toward changes in the cellular environment. Fission produces small spherical mitochondria, whereas fusion produces tubular or elongated mitochondria $[14,15]$. It is well established that mitochondrial dynamics play a crucial role in the quality control of mitochondria. Fusion of mitochondria that are reversibly damaged with healthy mitochondria can favor their functional repair. In addition, fusion can limit the accumulation of mtDNA mutations during aging $[14,15]$. However, when mitochondria incur irreversible damage, fission leads to elimination of the damaged organelles (Figure 2) [87]. Mitochondrial dynamics are regulated by several different GTPases. Mitofusins 1 and 2 (Mfn1 and Mfn2) and optic atrophy 1 (Opal) induce mitochondrial fusion in the outer and inner mitochondrial membranes, respectively [88, 89], while dynamin-related protein 1 (Drpl) is a cytoplasmic protein that assembles into rings surrounding the mitochondrial outer membrane, where it interacts with fission protein 1 (Fis1) to promote fission [90, 91]. As mentioned above, mitochondrial fusion and fission are required for the regulation of cardiac homeostasis and adaptation to stress $[14,15]$. Mice with cardiac Mfn2 deletion develop cardiac hypertrophy associated with systolic dysfunction and mitochondrial 


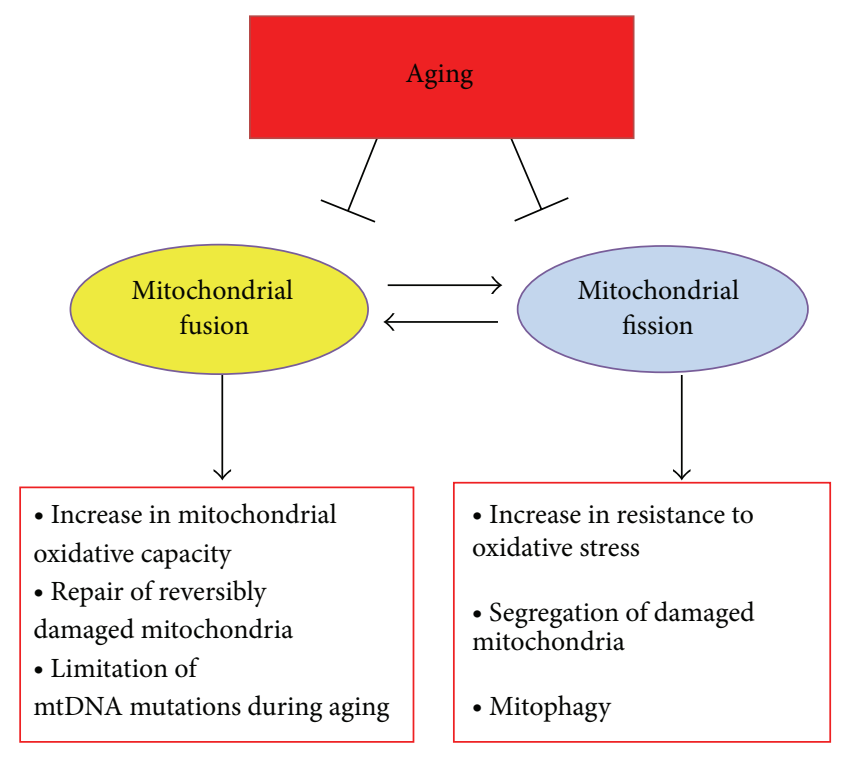

FIGURE 2: Mitochondrial dynamics. Schema explaining the main functions of mitochondrial fusion and fission.

dysfunction [92]. It was also found that Mfn2 mediates the differentiation of embryonal stem cells into cardiomyocytes and cardiac embryogenesis through calcineurin and Notch signaling [93]. On the other hand, Mfn2 overexpression promotes apoptosis in cardiomyocytes exposed to oxidative stress [94]. Mice with systemic heterozygous deletion of Opal develop pronounced hypertrophy and dysfunction in response to pressure overload [95]. Interestingly, inhibition of either Mfn1 or Mfn2 promotes generation of fragmented mitochondria, which inhibit cardiomyocyte mitochondrial transition pore opening and confer protection in response to oxidative stress $[92,96]$. This data indicates that integrity of mitochondrial fusion is required for cardiomyocyte homeostasis and maintenance of cardiac function at baseline conditions. However, during stress, accumulation of small mitochondria is protective against ROS accumulation in cardiomyocytes. Functional mitochondrial fission is also crucial for preservation of cardiac structure and function. In fact, cardiac-specific deletion of Drp1 is associated with the development of cardiac dilation and dysfunction over time, which is related to inhibition of mitochondrial fission and autophagy (Ikeda Y and Sadoshima J, unpublished data).

Mitochondrial fission is an essential requirement for mitophagy [97]. Inhibition of fission results in disruption of mitophagy and accumulation of dysfunctional mitochondria [87]. The fragments which result from fission of mitochondria can differ in membrane potential $(\Delta \psi \mathrm{m})$ and fragments with low $\Delta \psi \mathrm{m}$ are more likely to be targeted by mitophagy [87]. Oxidative stress can lead to this formation of asymmetrical daughter mitochondria characterized by different $\Delta \psi \mathrm{m}$ [87]. In addition, increased mitochondrial pore opening promotes mitophagy through cyclophilin-D-dependent mechanisms, as observed in cardiomyocytes [98].

Several specific proteins have been reported to play a key role in the molecular regulation of mitophagy. PTENinduced kinase 1 (PINK1) and parkin are associated with

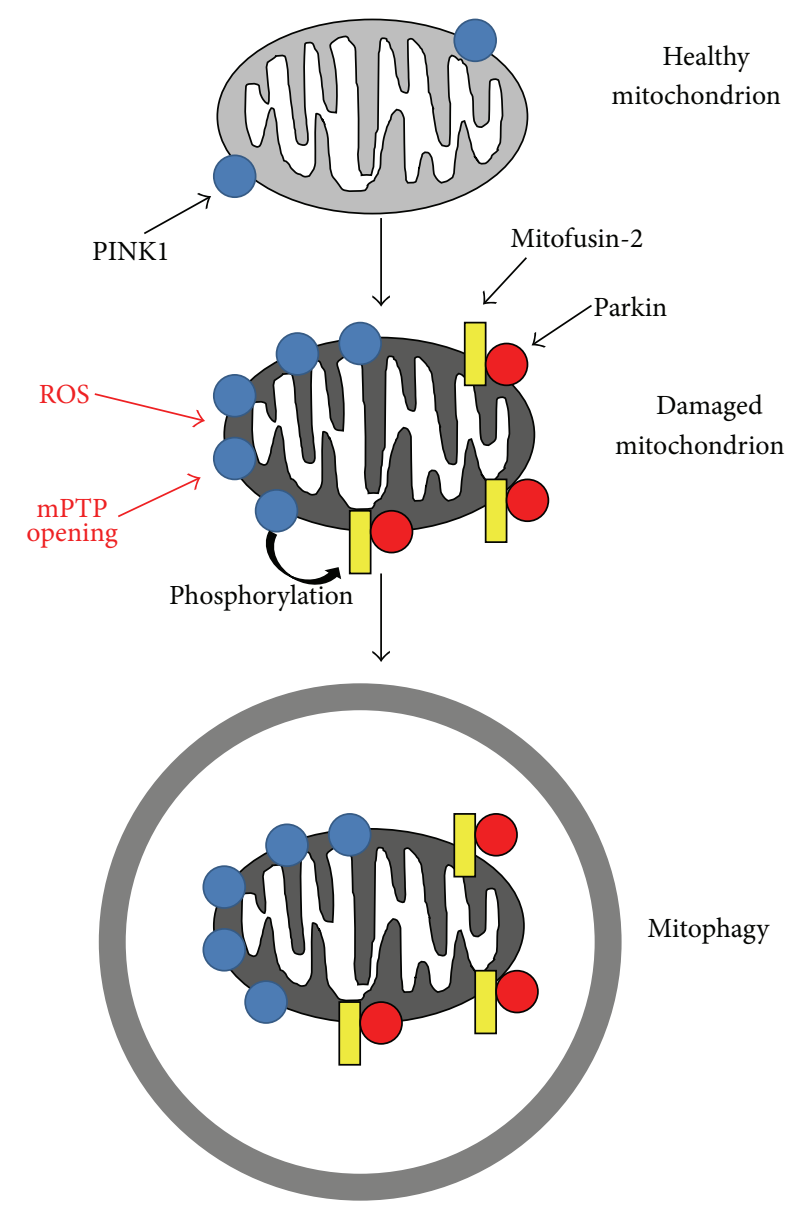

FIGURE 3: Mechanisms of mitophagy induction. Schema representing the main mechanisms of mitophagy induction. PINK1 accumulates in damaged mitochondria and phosphorylates mitofusin2. Phosphorylated mitofusin-2 recruits parkin, which ubiquitinates several mitochondrial targets. Mitochondrial ubiquitination by parkin promotes mitophagy.

familial Parkinson's disease and have recently been identified as important regulators of mitophagy [97]. PINK1 is a serine/threonine kinase that is localized in mitochondria. Upon mitochondrial damage and loss of inner mitochondrial membrane potential, PINK1 is stabilized, accumulates, and promotes the recruitment of parkin in damaged mitochondria [99]. Parkin is a cytosolic E3-ubiquitin ligase that accumulates in depolarized mitochondria and promotes their clearance by mitophagy [100]. Parkin ubiquitinates several targets in damaged mitochondria, including voltage-dependent anion channels (VDAC) and Mfn2, and recruits ubiquitinbinding deacetylase HDAC6 and p62/sequestosome-1, which interact with LC3 and promote assembly of the autophagosome and, ultimately, removal of the damaged mitochondria (Figure 3) [101-103]. Of note, double deletion of Mfnl and Mfn2 in the adult heart promotes cardiac dysfunction and mitochondrial fragmentation without associated mitophagy, suggesting that Mfn1 and Mfn2 are important for the mitophagic mechanism [104]. This hypothesis is supported by the recent demonstration that PINK1 phosphorylates Mfn2 
at threonine 111 and serine 442 to promote its binding with parkin in damaged mitochondria, ultimately leading to mitophagic removal [105]. Aside from demonstrating the mechanisms through which PINK1 tags damaged mitochondria for subsequent recognition by parkin, this study strongly supports the existence of a tight relationship between mitochondrial dynamics and mitophagy. Furthermore, parkin and mitofusins have been demonstrated to cooperate in a balanced manner to regulate mitophagy and mitochondrial spheroid formation in response to ROS accumulation [106]. In addition, Mfn2 is involved in the regulation of autophagosome-lysosome fusion in cardiomyocytes [107].

Other specific proteins that have been reported to play a role in mitophagy are $\mathrm{Bcl}-2$ /adenovirus E1B $19-\mathrm{kDa}-$ interacting protein-3 (Bnip3) and Nip3-like protein X (Nix) [97]. Both Nix and Bnip3 have been implicated in the pathogenesis of cancer and heart disease [108]. Nix is reported to be required for the selective elimination of mitochondria in erythrocytes in peripheral blood [109], while Bnip3 has been reported to induce hypoxia-mediated mitophagy downstream of hypoxia-inducible factor- $1 \alpha$ [110]. Bnip3 was also shown to promote translocation of Drpl in response to oxidative stress in cardiomyocytes [111], and Nix-dependent mitochondrial depolarization promotes mitophagy [108]. In addition, Nix interacts directly with LC3 and recruits the autophagosome to damaged mitochondria, thus acting as a receptor for autophagosome recruitment in this context [112].

Autophagic activity is reduced during aging [16]. As a direct consequence, mitophagy is also inhibited [16]. Mitochondrial dynamics are also altered during aging. Drpl and Mfn2 expression levels are reduced in aged skeletal muscle [113]. In addition, peroxisome proliferator-activated receptor gamma coactivator (PGC)-1 $\alpha$ levels are reduced in the skeletal muscle of the elderly [114]. PGC- $1 \alpha$ promotes mitochondrial biogenesis and fusion by upregulating the levels of Mfn2 in human skeletal muscle [115]. Fis1 overexpression reduces cellular senescence in hepatic cells, suggesting that activation of fission offsets the aging process [116]. Abnormalities in mitochondrial dynamics and mitophagy significantly contribute to the accumulation of aberrant and dysfunctional mitochondria with progressive accumulation of mtDNA mutations.

Therefore, mitophagy is critical for turnover of whole damaged mitochondria. However, other cellular mechanisms promoting degradation of damaged mitochondrial proteins are also crucial for mitochondrial quality control [117, 118]. Damaged and misfolded mitochondrial proteins can be cleared by mitochondrial chaperones and proteases $[117,118]$. Accumulating lines of evidence also indicate that the ubiquitin-proteasome system is critical for regulation of mitochondrial quality control $[117,118]$. Inhibition of this system results in the accumulation of damaged and dysfunctional mitochondria [119]. In addition, defects of the ubiquitin-proteasome system result in cardiac hypertrophy and dysfunction and defective cardiac responses to stress $[120,121]$. Multiple mitochondrial proteins have been shown to undergo ubiquitination and accumulate when proteasome is inhibited. Upon mitochondrial damage, parkin was shown to mediate the proteasomal degradation of $\mathrm{Mfn} 1$ and 2
[122]. VDAC and Tom 20 were also found to be potential targets of the ubiquitin-proteasome system [122]. Intriguingly, proteasomal degradation appears to also regulate the turnover of the inner mitochondrial membrane uncoupling protein 2 [123] and of the mitochondrial matrix oligomycin sensitivity conferral protein (OSCP) [124]. This data suggests the existence of complex mechanisms exposing the damaged inner membrane and matrix proteins to the ubiquitinproteasome system. Interestingly, proteasomal degradation of mitochondrial proteins was shown to be tightly connected to the regulation of mitochondrial dynamics and mitophagy [117, 118]. Mfn1, Drp-1, and Fis1 can be degraded by the ubiquitin-proteasome system, thereby affecting fusion and fission [122, 125-127]. As described above, mitochondrial protein ubiquitination by parkin is required for mitophagy induction [122, 125, 127]. Intriguingly, it has been recently demonstrated that proteins regulating proteasome activity are also deeply involved in the regulation of the autophagic process [128]. In addition, a cross-talk between ubiquitinproteasome system and autophagy has been demonstrated in cardiomyocytes in vitro and in vivo [129]. This data suggests an interplay between the ubiquitin-proteasome system and autophagy, which may share common mechanisms of regulation.

\section{The Role of Autophagy in Cardiac Ischemia, Remodeling, and Hypertrophy}

Cardiac aging is associated with increased susceptibility to ischemic injury and remodeling $[45,46]$. Autophagy and mitochondrial dynamics play an important role in regulating cardiac adaptation to stress $[14,15,58,59]$. Therefore, agedependent abnormalities in autophagy and mitochondrial dynamics might be involved in the increased fragility of the aged heart during stress.

Cardiac ischemia decreases cellular ATP content, which leads to energy stress and abnormal ROS production associated with mitochondrial dysfunction [130]. Autophagy activation in the ischemic heart protectively counterbalances these mechanisms $[16-18,65]$. Our group has recently elucidated the mechanisms through which autophagy is upregulated during energy deprivation. We found that activation of AMPK [131] and glycogen synthase kinase (GSK)-3 $\beta$ [132] as well as inhibition of Ras homolog enriched in brain (Rheb) [133] contributes to autophagy activation through the inhibition of mTORC1. Nox4-dependent production of ROS in the endoplasmic reticulum is also an important signaling event for induction of autophagy during energy deprivation through the activation of PERK signaling [24]. In addition, starvation activates FOXO1 through SIRT1mediated deacetylation, which induces expression of Rab7 and stimulates autolysosome formation and autophagic flux [134].

Autophagy inhibition increases ischemic injury. Inhibition of endogenous AMPK during prolonged ischemia causes suppression of autophagy, accompanied by enlargement of the myocardial infarct [131]. Similarly, inhibition of GSK-3 $\beta$ and activation of Rheb enhance ischemic 
injury during ischemia $[132,133]$. Interestingly, the increased myocardial susceptibility to ischemia associated with obesity and metabolic syndrome involves a deregulated activation of the $\mathrm{Rheb} / \mathrm{mTORC1}$ pathway $[67,133]$.

The energy crisis caused by ischemia in the heart is at least partially resolved at the time of reperfusion, which further activates autophagy through mechanisms different from those involved in autophagy during ischemia [131]. We have reported that ROS generated in the mouse heart during reperfusion injury mediate the upregulation of Beclin1 [131]. In these experiments, Beclinl was strongly upregulated during the reperfusion phase but not during the ischemic phase. These results suggest that strong induction of Beclin1 by ROS may play an important role in mediating the stimulation of autophagy during reperfusion.

Whether autophagy induced during reperfusion is beneficial or detrimental remains controversial. Although Hamacher-Brady et al. [135] showed that enhancing autophagic flux during ischemia/reperfusion protects against ischemic/reperfusion injury in cardiomyocytes, we found that autophagosome formation and cardiac ischemic injury are significantly attenuated in mice with heterozygous disruption of Beclin1 [131] and GSK-3 $\beta$ [132]. It is possible that exaggerated activation of autophagy during excessive ROS production may trigger cell death. In this regard, it has been recently proved the existence of a form of cell death named "autosis," which is characterized by cellular nuclear convolution at early stages and by focal swelling of perinuclear space at late stages and which is blocked by autophagy inhibition or knockdown of the $\mathrm{Na}+/ \mathrm{K}+$-ATPase $\alpha 1$ subunit, but not by inhibitors of apoptosis or necrosis [136].

Despite the controversy of whether autophagy activation is protective or detrimental during reperfusion injury, mitophagy activation appears to protect the heart during ischemia/reperfusion. Inhibition of Drpl was found to promote ischemic injury in the heart through inhibition of fission and mitophagy (Ikeda Y and Sadoshima J, unpublished observations). Parkin inhibition increases cardiomyocyte death in response to hypoxia-reoxygenation in vitro, and parkin deletion in vivo abolished the cardioprotective effect of ischemic preconditioning [137]. In addition, simvastatin was shown to reduce cardiac damage in response to ischemia/reperfusion by activating mitophagy [138].

Autophagy inhibition negatively impacts cardiac remodeling after chronic myocardial infarction due to accumulation of misfolded proteins and mitochondrial dysfunction. We recently found that the serine/threonine kinase MST1 phosphorylates Beclinl at threonine 108, thereby promoting its interaction with $\mathrm{Bcl}-2$ and inhibiting autophagy. MST1-dependent inhibition of autophagy accelerates cardiac remodeling during chronic myocardial infarction [139]. Interestingly, parkin knockout mice display reduced mitophagy and survival after myocardial infarction, together with increased cardiac remodeling and accumulation of dysfunctional mitochondria [140]. These data suggest that mitophagy plays a crucial role in delaying cardiac remodeling after myocardial infarction.
Aging is associated with increased cardiac hypertrophy, and defects in autophagy promote cardiac hypertrophy [58, 59]. Cardiac deletion of Atg5 is associated with the development of eccentric hypertrophy over time [76]. We recently reported an experiment in which mice were subjected to transverse aortic constriction (TAC) for 1 week, after which the constriction was removed (DeTAC) [141]. Regression of cardiac hypertrophy was observed after DeTAC, accompanied by upregulation of autophagy in conjunction with upregulation of FOXO1. This regression of cardiac hypertrophy was significantly attenuated by inhibition of either autophagy or FOXO1. These results suggest that autophagy and FOXO1 play an essential role in mediating the regression of cardiac hypertrophy during mechanical unloading [141]. In addition, PINK1 knockout mice develop cardiac dysfunction and hypertrophy associated with oxidative stress, mitochondrial dysfunction, fibrosis, and apoptosis at baseline [142], indicating that mitophagy is important for inhibiting maladaptive hypertrophy. Interestingly, in Drosophila, inhibition of parkin leads to progressive cardiac dilation and dysfunction through inhibition of mitophagy, increased mitochondrial fusion and subsequent contagion of healthy mitochondria with damaged ones [143].

\section{Perspectives}

Translational research represents a stem of scientific research that helps make findings from basic science useful for practical applications that enhance human health and wellbeing. Deeply established with multidisciplinary collaboration, translational research has the enormous potential to move applied science forward. To date, this is particularly true in so-called "translational medicine" research that aims to move "from bench to bedside" or from laboratory experiments through clinical trials to point-of-care patient applications. In this context, mitochondrial autophagy and mitochondrial dynamics emerge to have an important role in regulating mitochondrial function and quality control. However, autophagy appears to be inhibited during aging [16]. In future studies, it will be important to investigate the relationship between aging and mitophagy and mitochondrial dynamics more deeply. In particular, it should be interesting to evaluate the molecular mechanisms and signaling pathway interactions through which aging affects mitophagy, mitochondrial fusion, and mitochondrial fission. This information would help in the generation of specific drugs that are capable of reactivating these mechanisms to limit age-dependent mitochondrial dysfunction and ROS accumulation. In support of the potential usefulness of reactivating mitophagy to increase longevity, it has been recently demonstrated that parkin overexpression extends life span in Drosophila [144]. Similar studies in mammals are strongly encouraged.

Until specific pharmacological agents to target abnormalities in mitophagy and mitochondrial dynamics during aging are identified, the efficacy of reactivating general autophagy should be tested [19]. Autophagy would eliminate intracellular waste material and dysfunctional mitochondria that cause abnormal ROS generation during aging and cardiac derangements (Figure 4). Several interventions are known to induce 


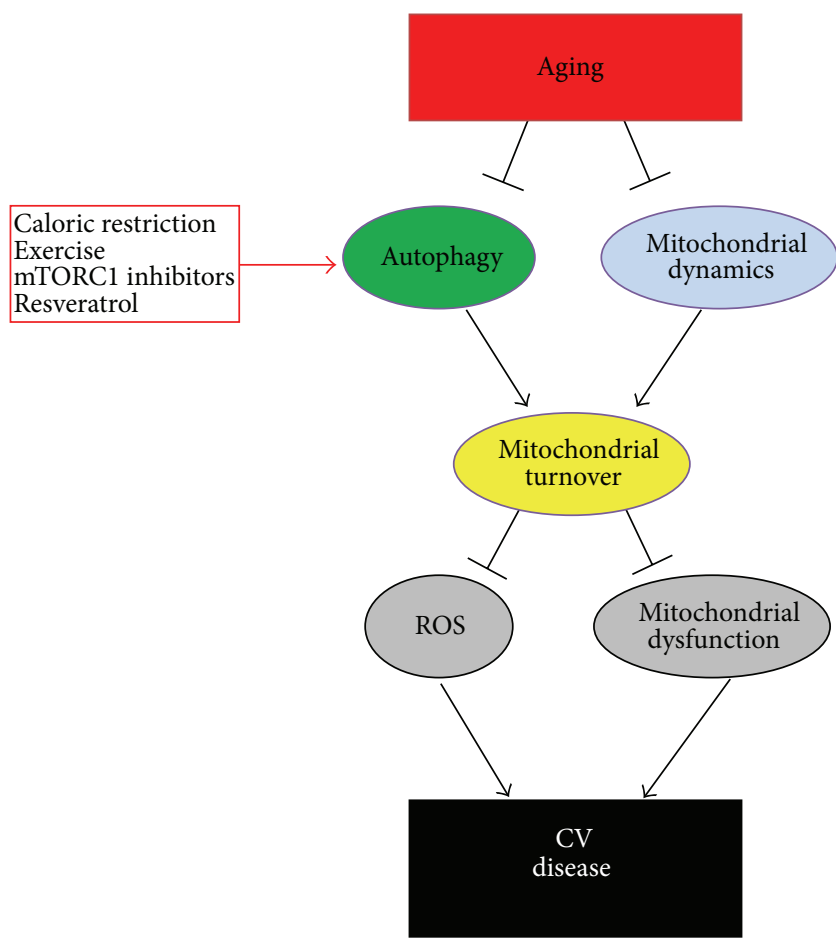

FIgURE 4: The importance of autophagy in the aged heart. Aging inhibits autophagy and affects mitochondrial dynamics. Autophagy inhibition is associated with accumulation of damaged mitochondria and oxidative stress, which favor the development of cardiovascular diseases.

autophagy and could be useful for promoting longevity. Caloric restriction, defined as a reduction in food intake without malnutrition, is a well-known antiaging intervention and physiological inducer of autophagy $[16,78]$. Long-term caloric restriction inhibits the age-associated decline in diastolic function in the heart [84]. Pharmacological induction of autophagy may also effectively increase longevity. Metformin is an activator of AMPK that promotes autophagy, which contributes to its antidiabetic effect [19]. Of note, lowdose metformin was recently found to extend health span and life span in mice without metabolic disorders [145, 146]. Metformin administration increases insulin sensitivity, reduces cholesterol levels, improves physical performance, and exerts antioxidant and anti-inflammatory effects during the aging process [145]. Suppression of mTOR by rapamycin prevents cardiac senescence in conjunction with inhibition of lipofuscin accumulation in the heart $[83,85]$, and resveratrol is able to induce autophagy and increase longevity [147]. Exercise also induces autophagy in skeletal muscle, which contributes to the improvement in glucose tolerance induced by exercise in the context of a high-fat diet [148]. The actual role of autophagy in mediating the beneficial effects of these compounds on life span still requires clarification. Of note, excessive activation of autophagy would not necessarily be protective because it could cause depletion of essential proteins and organelles [19, 67-69]. Future studies should therefore also investigate the level to which it would be beneficial to reactivate autophagy in the aged heart. Notably, all these emerging data have obvious and nonnegligible theoretical and applicative implications: they start from a biological/molecular core that is made of novel observations, concepts, and tools and aim to reach wed cell biology with clinical connotations. Accordingly, based on this new view and taken altogether, these advances in cell biology may herald a new area of cardiovascular regenerative antiaging and personalized medicine in the upcoming years, exploiting this body of evidence as a long-missed benchmark for the development of approaches moving from insight to insight and back.

\section{Conflict of Interests}

The authors declare that there is no conflict of interests regarding the publication of this paper.

\section{Acknowledgments}

The authors wish to thank Daniela K. Zablocki and Christopher D. Brady for critical reading of the paper and suggestions. This work was supported in part by U.S. Public Health Service Grants HL102738, HL67724, HL69020, HL91469, AG23039, and AG27211. This work was also supported by the Fondation Leducq Transatlantic Networks of Excellence. Sebastiano Sciarretta has been supported by a Postdoctoral Fellowship from the Founders Affiliate, American Heart Association, and partially by a grant from the Italian Society of Cardiology and Italian Society of Hypertension.

\section{References}

[1] W. Lutz, W. Sanderson, and S. Scherbov, "The coming acceleration of global population ageing," Nature, vol. 451, pp. 716-719, 2008.

[2] A. B. Newman, A. M. Arnold, B. L. Naydeck et al., "“'Successful Aging": effect of subclinical cardiovascular disease," Archives of Internal Medicine, vol. 163, no. 19, pp. 2315-2322, 2003.

[3] D. Lloyd-Jones, R. Adams, M. Carnethon et al., "Heart disease and stroke statistics-2009 update: a report from the American Heart Association Statistics Committee and Stroke Statistics Subcommittee," Circulation, vol. 119, no. 3, pp. e21-e181, 2009.

[4] A. Buchberger, B. Bukau, and T. Sommer, "Protein quality control in the cytosol and the endoplasmic reticulum: brothers in arms," Molecular Cell, vol. 40, no. 2, pp. 238-252, 2010.

[5] M. S. Denzel, N. J. Storm, A. Gutschmidt et al., "Hexosamine pathway metabolites enhance protein quality control and prolong life," Cell, vol. 156, pp. 1167-1178, 2014.

[6] T. Finkel, M. Serrano, and M. A. Blasco, "The common biology of cancer and ageing," Nature, vol. 448, no. 7155, pp. 767-774, 2007.

[7] D. Harman, “The biologic clock: the mitochondria?” Journal of the American Geriatrics Society, vol. 20, no. 4, pp. 145-147, 1972.

[8] H. J. Harmon, S. Nank, and R. A. Floyd, "Age-dependent changes in rat brain mitochondria of synaptic and non-synaptic origins," Mechanisms of Ageing and Development, vol. 38, no. 2, pp. 167-177, 1987.

[9] T. Finkel and N. J. Holbrook, "Oxidants, oxidative stress and the biology of ageing," Nature, vol. 408, pp. 239-247, 2000. 
[10] D. Dutta, R. Calvani, R. Bernabei, C. Leeuwenburgh, and E. Marzetti, "Contribution of impaired mitochondrial autophagy to cardiac aging: mechanisms and therapeutic opportunities," Circulation Research, vol. 110, no. 8, pp. 1125-1138, 2012.

[11] T. Lu and T. Finkel, "Free radicals and senescence," Experimental Cell Research, vol. 314, no. 9, pp. 1918-1922, 2008.

[12] O. Bergmann, R. D. Bhardwaj, S. Bernard et al., "Evidence for cardiomyocyte renewal in humans," Science, vol. 324, no. 5923, pp. 98-102, 2009.

[13] X. Wang, H. Su, and M. J. Ranek, "Protein quality control and degradation in cardiomyocytes," Journal of Molecular and Cellular Cardiology, vol. 45, no. 1, pp. 11-27, 2008.

[14] R. J. Youle and A. M. van der Bliek, "Mitochondrial fission, fusion, and stress," Science, vol. 337, no. 6098, pp. 1062-1065, 2012.

[15] A. Y. Seo, A. M. Joseph, D. Dutta, J. C. Y. Hwang, J. P. Aris, and C. Leeuwenburgh, "New insights into the role of mitochondria in aging: mitochondrial dynamics and more," Journal of Cell Science, vol. 123, no. 15, pp. 2533-2542, 2010.

[16] D. C. Rubinsztein, G. Mariño, and G. Kroemer, "Autophagy and aging," Cell, vol. 146, no. 5, pp. 682-695, 2011.

[17] K. Wang and D. J. Klionsky, "Mitochondria removal by autophagy," Autophagy, vol. 7, no. 3, pp. 297-300, 2011.

[18] B. Levine and G. Kroemer, "Autophagy in the pathogenesis of disease," Cell, vol. 132, no. 1, pp. 27-42, 2008.

[19] S. Sciarretta, P. Zhai, M. Volpe, and J. Sadoshima, "Pharmacological modulation of autophagy during cardiac stress," Journal of Cardiovascular Pharmacology, vol. 60, no. 3, pp. 235-241, 2012.

[20] J. Ruef, K. Peter, T. K. Nordt, M. S. Runge, W. Kubler, and C. Bode, "Oxidative stress and atherosclerosis: its relationship to growth factors, thrombus formation and therapeutic approaches," Thrombosis and Haemostasis, vol. 82, supplement 1, pp. 32-37, 1999.

[21] L. J. Marnett, “Oxyradicals and DNA damage," Carcinogenesis, vol. 21, no. 3, pp. 361-370, 2000.

[22] H. Misonou, M. Morishima-Kawashima, and Y. Ihara, "Oxidative stress induces intracellular accumulation of amyloid $\beta$ protein $(\mathrm{A} \beta)$ in human neuroblastoma cells," Biochemistry, vol. 39, no. 23, pp. 6951-6959, 2000.

[23] T. Finkel, "Signal transduction by reactive oxygen species," Journal of Cell Biology, vol. 194, no. 1, pp. 7-15, 2011.

[24] S. Sciarretta, P. Zhai, D. Shao et al., "Activation of NADPH oxidase 4 in the endoplasmic reticulum promotes cardiomyocyte autophagy and survival during energy stress through the protein kinase RNA-activated-like endoplasmic reticulum kinase/eukaryotic initiation factor 2alpha/activating transcription factor 4 pathway," Circulation Research, vol. 113, pp. 12531264, 2013.

[25] M. Holzenberger, J. Dupont, B. Ducos et al., "IGF-1 receptor regulates lifespan and resistance to oxidative stress in mice," Nature, vol. 421, no. 6919, pp. 182-187, 2003.

[26] T. Ago, J. Kuroda, J. Pain, C. Fu, H. Li, and J. Sadoshima, "Upregulation of Nox4 by hypertrophic stimuli promotes apoptosis and mitochondrial dysfunction in cardiac myocytes," Circulation Research, vol. 106, no. 7, pp. 1253-1264, 2010.

[27] J. E. Leadsham, G. Sanders, S. Giannaki et al., "Loss of cytochrome $c$ oxidase promotes RAS-dependent ROS production from the ER resident NADPH oxidase, Ynolp, in yeast," Cell Metabolism, vol. 18, no. 2, pp. 279-286, 2013.
[28] E. Migliaccio, M. Giogio, S. Mele et al., "The $p 66^{\text {shc }}$ adaptor protein controls oxidative stress response and life span in mammals," Nature, vol. 402, no. 6759, pp. 309-313, 1999.

[29] G. Martínez-Sánchez, A. Giuliani, G. Pérez-Davison, and O. S. León-Fernández, "Oxidized proteins and their contribution to redox homeostasis," Redox Report, vol. 10, no. 4, pp. 175-185, 2005.

[30] J. E. Nuss, K. B. Choksi, J. H. DeFord, and J. Papaconstantinou, "Decreased enzyme activities of chaperones PDI and BiP in aged mouse livers," Biochemical and Biophysical Research Communications, vol. 365, no. 2, pp. 355-361, 2008.

[31] A. Vasilaki and M. J. Jackson, "Role of reactive oxygen species in the defective regeneration seen in aging muscle," Free Radical Biology and Medicine, vol. 65, pp. 317-323, 2013.

[32] R. Tao, M. C. Coleman, J. D. Pennington et al., "Sirt3-mediated deacetylation of evolutionarily conserved lysine 122 regulates MnSOD activity in response to stress," Molecular Cell, vol. 40, no. 6, pp. 893-904, 2010.

[33] S. Someya, W. Yu, W. C. Hallows et al., "Sirt3 mediates reduction of oxidative damage and prevention of age-related hearing loss under caloric restriction," Cell, vol. 143, no. 5, pp. 802-812, 2010.

[34] A. Brunet, L. B. Sweeney, J. F. Sturgill et al., "Stress-Dependent Regulation of FOXO Transcription Factors by the SIRT1 Deacetylase," Science, vol. 303, no. 5666, pp. 2011-2015, 2004.

[35] O. M. Palacios, J. J. Carmona, S. Michan et al., "Diet and exercise signals regulate SIRT3 and activate AMPK and PGC-1alpha in skeletal muscle," Aging, vol. 1, no. 9, pp. 771-783, 2009.

[36] R. R. Alcendor, S. Gao, P. Zhai et al., "Sirtl regulates aging and resistance to oxidative stress in the heart," Circulation Research, vol. 100, no. 10, pp. 1512-1521, 2007.

[37] S. Caito, S. Rajendrasozhan, S. Cook et al., "SIRT1 is a redoxsensitive deacetylase that is post-translationally modified by oxidants and carbonyl stress," The FASEB Journal, vol. 24, no. 9, pp. 3145-3159, 2010.

[38] A. P. Gomes, N. L. Price, A. J. Ling, J. J. Moslehi, M. K. Montgomery, and L. Rajman, "Declining $\mathrm{NAD}^{+}$induces a pseudohypoxic state disrupting nuclear-mitochondrial communication during aging," Cell, vol. 155, pp. 1624-1638, 2013.

[39] X. Li, J. Song, L. Zhang et al., "Activation of the AMPK-FOXO3 pathway reduces fatty acid-induced increase in intracellular reactive oxygen species by upregulating thioredoxin," Diabetes, vol. 58, no. 10, pp. 2246-2257, 2009.

[40] D. Shao, S. Oka, T. Liu et al., "A redox-dependent mechanism for regulation of AMPK activation by Thioredoxinl during energy starvation," Cell Metabolism, vol. 19, no. 2, pp. 232-245, 2014.

[41] K. Zarse, S. Schmeisser, M. Groth et al., "Impaired insulin/IGF1 signaling extends life span by promoting mitochondrial Lproline catabolism to induce a transient ROS signal," Cell Metabolism, vol. 15, no. 4, pp. 451-465, 2012.

[42] J. M. van Raamsdonk and S. Hekimi, "Deletion of the mitochondrial superoxide dismutase sod-2 extends lifespan in Caenorhabditis elegans," PLoS Genetics, vol. 5, no. 2, Article ID e1000361, 2009.

[43] D. C. Chan, "Mitochondria: dynamic organelles in disease, aging, and development," Cell, vol. 125, no. 7, pp. 1241-1252, 2006.

[44] V. W. S. Liu, C. Zhang, and P. Nagley, "Mutations in mitochondrial DNA accumulate differentially in three different human tissues during ageing," Nucleic Acids Research, vol. 26, no. 5, pp. 1268-1275, 1998. 
[45] S. A. Mohamed, T. Hanke, A. W. Erasmi et al., "Mitochondrial DNA deletions and the aging heart," Experimental Gerontology, vol. 41, no. 5, pp. 508-517, 2006.

[46] W. K. Porteous, A. M. James, P. W. Sheard et al., "Bioenergetic consequences of accumulating the common 4977-bp mitochondrial DNA deletion," European Journal of Biochemistry, vol. 257, no. 1, pp. 192-201, 1998.

[47] H. P. Indo, M. Davidson, H. Yen et al., "Evidence of ROS generation by mitochondria in cells with impaired electron transport chain and mitochondrial DNA damage," Mitochondrion, vol. 7, no. 1-2, pp. 106-118, 2007.

[48] A. Trifunovic, A. Wredenberg, M. Falkenberg et al., "Premature ageing in mice expressing defective mitochondrial DNA polymerase," Nature, vol. 429, no. 6990, pp. 417-423, 2004.

[49] D. Han, R. Canali, J. Garcia, R. Aguilera, T. K. Gallaher, and E. Cadenas, "Sites and mechanisms of aconitase inactivation by peroxynitrite: modulation by citrate and glutathione," Biochemistry, vol. 44, no. 36, pp. 11986-11996, 2005.

[50] J. R. Veatch, M. A. McMurray, Z. W. Nelson, and D. E. Gottschling, "Mitochondrial dysfunction leads to nuclear genome instability via an iron-sulfur cluster defect," Cell, vol. 137, no. 7, pp. 1247-1258, 2009.

[51] J. Nunnari and A. Suomalainen, "Mitochondria: in sickness and in health," Cell, vol. 148, no. 6, pp. 1145-1159, 2012.

[52] S. E. Schriner, N. J. Linford, G. M. Martin et al., "Medecine: Extension of murine life span by overexpression of catalase targeted to mitochondria," Science, vol. 308, no. 5730, pp. 19091911, 2005.

[53] H. Y. Lee, C. S. Choi, A. L. Birkenfeld et al., "Targeted expression of catalase to mitochondria prevents age-associated reductions in mitochondrial function and insulin resistance," Cell Metabolism, vol. 12, no. 6, pp. 668-674, 2010.

[54] V. I. Pérez, H. van Remmen, A. Bokov, C. J. Epstein, J. Vijg, and A. Richardson, "The overexpression of major antioxidant enzymes does not extend the lifespan of mice," Aging Cell, vol. 8, no. 1, pp. 73-75, 2009.

[55] V. I. Pérez, A. Bokov, H. V. Remmen et al., "Is the oxidative stress theory of aging dead?" Biochimica et Biophysica Acta, vol. 1790, no. 10, pp. 1005-1014, 2009.

[56] B. H. Ahn, H. S. Kim, S. Song et al., "A role for the mitochondrial deacetylase Sirt3 in regulating energy homeostasis," Proceedings of the National Academy of Sciences of the United States of America, vol. 105, no. 38, pp. 14447-14452, 2008.

[57] A. V. Hafner, J. Dai, A. P. Gomes et al., "Regulation of the mPTP by SIRT3-mediated deacetylation of CypD at lysine 166 suppresses age-related cardiac hypertrophy," Aging, vol. 2, no. 12, pp. 914-923, 2010.

[58] D. Dai, P. S. Rabinovitch, and Z. Ungvari, "Mitochondria and cardiovascular aging," Circulation Research, vol. 110, no. 8, pp. 1109-1124, 2012.

[59] D. Dai and P. S. Rabinovitch, "Cardiac aging in mice and humans: the role of mitochondrial oxidative stress," Trends in Cardiovascular Medicine, vol. 19, no. 7, pp. 213-220, 2009.

[60] T. Ago, T. Liu, P. Zhai et al., "A redox-dependent pathway for regulating class II HDACs and cardiac hypertrophy," Cell, vol. 133, no. 6, pp. 978-993, 2008.

[61] S. Sciarretta, F. Paneni, F. Palano et al., "Role of the reninangiotensin-aldosterone system and inflammatory processes in the development and progression of diastolic dysfunction," Clinical Science, vol. 116, no. 6, pp. 467-477, 2009.
[62] F. Qin, D. A. Siwik, S. Lancel, J. Zhang, G. M. Kuster, and I. Luptak, "Hydrogen peroxide-mediated SERCA cysteine 674 oxidation contributes to impaired cardiac myocyte relaxation in senescent mouse heart," Journal of the American Heart Association, vol. 2, article e000184, 2013.

[63] J. S. Ingwall and R. G. Weiss, "Is the failing heart energy starved? On using chemical energy to support cardiac function," Circulation Research, vol. 95, no. 2, pp. 135-145, 2004.

[64] S. Sciarretta, F. Paneni, G. M. Ciavarella et al., "Evaluation of systolic properties in hypertensive patients with different degrees of diastolic dysfunction and normal ejection fraction," The American Journal of Hypertension, vol. 22, no. 4, pp. 437443, 2009.

[65] D. R. Green, L. Galluzzi, and G. Kroemer, "Mitochondria and the autophagy-inflammation-cell death axis in organismal aging," Science, vol. 333, no. 6046, pp. 1109-1112, 2011.

[66] T. Yorimitsu and D. J. Klionsky, "Autophagy: molecular machinery for self-eating," Cell Death and Differentiation, vol. 12, supplement 2, pp. 1542-1552, 2005.

[67] S. Sciarretta, M. Volpe, and J. Sadoshima, "Is reactivation of autophagy a possible therapeutic solution for obesity and metabolic syndrome?" Autophagy, vol. 8, no. 8, pp. 1252-1254, 2012.

[68] S. Sciarretta, D. Yee, V. Shenoy, N. Nagarajan, and J. Sadoshima, "The importance of autophagy in cardioprotection," High Blood Pressure \& Cardiovascular Prevention, vol. 21, no. 1, pp. 21-28, 2014.

[69] S. Sciarretta, M. Volpe, and J. Sadoshima, "NOX4 regulates autophagy during energy deprivation," Autophagy, vol. 10, no. 4, pp. 699-701, 2014.

[70] M. L. Tóth, T. Sigmond, É. Borsos et al., "Longevity pathways converge on autophagy genes to regulate life span in Caenorhabditis elegans," Autophagy, vol. 4, no. 3, pp. 330-338, 2008.

[71] M. Komatsu, S. Waguri, M. Koike et al., "Homeostatic levels of p62 control cytoplasmic inclusion body formation in autophagy-deficient mice," Cell, vol. 131, no. 6, pp. 1149-1163, 2007.

[72] M. Komatsu, S. Waguri, T. Ueno et al., "Impairment of starvation-induced and constitutive autophagy in Atg7deficient mice," Journal of Cell Biology, vol. 169, no. 3, pp. 425-434, 2005.

[73] M. Komatsu, S. Waguri, T. Chiba et al., "Loss of autophagy in the central nervous system causes neurodegeneration in mice," Nature, vol. 441, no. 7095, pp. 880-884, 2006.

[74] M. Komatsu, J. W. Qing, G. R. Holstein et al., "Essential role for autophagy protein Atg7 in the maintenance of axonal homeostasis and the prevention of axonal degeneration," Proceedings of the National Academy of Sciences of the United States of America, vol. 104, no. 36, pp. 14489-14494, 2007.

[75] H. S. Jung, K. W. Chung, J. Won Kim et al., "Loss of autophagy diminishes pancreatic beta cell mass and function with resultant hyperglycemia," Cell Metabolism, vol. 8, no. 4, pp. 318-324, 2008.

[76] M. Taneike, O. Yamaguchi, A. Nakai et al., "Inhibition of autophagy in the heart induces age-related cardiomyopathy," Autophagy, vol. 6, no. 5, pp. 600-606, 2010.

[77] D. E. Harrison, R. Strong, Z. D. Sharp et al., "Rapamycin fed late in life extends lifespan in genetically heterogeneous mice," Nature, vol. 460, no. 7253, pp. 392-395, 2009.

[78] R. J. Colman, R. M. Anderson, S. C. Johnson et al., "Caloric restriction delays disease onset and mortality in rhesus monkeys," Science, vol. 325, no. 5937, pp. 201-204, 2009. 
[79] I. Bjedov, J. M. Toivonen, F. Kerr et al., "Mechanisms of life span extension by rapamycin in the fruit fly Drosophila melanogaster," Cell Metabolism, vol. 11, no. 1, pp. 35-46, 2010.

[80] A. Terman and U. T. Brunk, "Lipofuscin: mechanisms of formation and increase with age," Acta Pathologica, Microbiologica, et Immunologica Scandinavica, vol. 106, no. 2, pp. 265-276, 1998.

[81] T. Jung, N. Bader, and T. Grune, "Lipofuscin: formation, distribution, and metabolic consequences," Annals of the New York Academy of Sciences, vol. 1119, no. 1, pp. 97-111, 2007.

[82] C. Zhang and A. M. Cuervo, "Restoration of chaperonemediated autophagy in aging liver improves cellular maintenance and hepatic function," Nature Medicine, vol. 14, no. 9, pp. 959-965, 2008.

[83] J. M. Flynn, M. N. O’Leary, C. A. Zambataro et al., "Late-life rapamycin treatment reverses age-related heart dysfunction," Aging Cell, vol. 12, pp. 851-862, 2013.

[84] I. Ahmet, H. Tae, R. de Cabo, E. G. Lakatta, and M. I. Talan, "Effects of calorie restriction on cardioprotection and cardiovascular health," Journal of Molecular and Cellular Cardiology, vol. 51, no. 2, pp. 263-271, 2011.

[85] S. Sciarretta, M. Volpe, and J. Sadoshima, "Mammalian target of rapamycin signaling in cardiac physiology and disease," Circulation Research, vol. 114, no. 3, pp. 549-564, 2014.

[86] D. F. Dai, P. P. Karunadharma, Y. A. Chiao, N. Basisty, D. Crispin, and E. J. Hsieh, "Altered proteome turnover and remodeling by short-term caloric restriction or rapamycin rejuvenate the aging heart," Aging Cell, vol. 13, pp. 529-539, 2014.

[87] G. Twig, A. Elorza, A. J. A. Molina et al., "Fission and selective fusion govern mitochondrial segregation and elimination by autophagy," The EMBO Journal, vol. 27, no. 2, pp. 433-446, 2008.

[88] T. Koshiba, S. A. Detmer, J. T. Kaiser, H. Chen, J. M. McCaffery, and D. C. Chan, "Structural basis of mitochondrial tethering by mitofusin complexes," Science, vol. 305, no. 5685, pp. 858-862, 2004.

[89] S. Cipolat, O. M. De Brito, B. Dal Zilio, and L. Scorrano, "OPA1 requires mitofusin 1 to promote mitochondrial fusion," Proceedings of the National Academy of Sciences of the United States of America, vol. 101, no. 45, pp. 15927-15932, 2004.

[90] E. Smirnova, L. Griparic, D.-L. Shurland, and A. M. van der Bliek, "Dynamin-related protein Drpl is required for mitochondrial division in mammalian cells," Molecular Biology of the Cell, vol. 12, no. 8, pp. 2245-2256, 2001.

[91] Y. Yoon, E. W. Krueger, B. J. Oswald, and M. A. McNiven, “The mitochondrial protein hFisl regulates mitochondrial fission in mammalian cells through an interaction with the dynamin-like protein DLP1," Molecular and Cellular Biology, vol. 23, no. 15, pp. 5409-5420, 2003.

[92] K. N. Papanicolaou, R. J. Khairallah, G. A. Ngoh et al., "Mitofusin-2 maintains mitochondrial structure and contributes to stress-induced permeability transition in cardiac myocytes," Molecular and Cellular Biology, vol. 31, no. 6, pp. 1309-1328, 2011.

[93] A. Kasahara, S. Cipolat, Y. Chen, G. W. Dorn II., and L. Scorrano, "Mitochondrial fusion directs cardiomyocyte differentiation via calcineurin and Notch signaling," Science, vol. 342, no. 6159, pp. 734-737, 2013.

[94] T. Shen, M. Zheng, C. Cao et al., "Mitofusin-2 is a major determinant of oxidative stress-mediated heart muscle cell apoptosis," Journal of Biological Chemistry, vol. 282, no. 32, pp. 23354-23361, 2007.
[95] J. Piquereau, F. Caffin, M. Novotova et al., "Down-regulation of OPA1 alters mouse mitochondrial morphology, PTP function, and cardiac adaptation to pressure overload," Cardiovascular Research, vol. 94, no. 3, pp. 408-417, 2012.

[96] K. N. Papanicolaou, G. A. Ngoh, E. R. Dabkowski et al., "Cardiomyocyte deletion of mitofusin-1 leads to mitochondrial fragmentation and improves tolerance to ROS-induced mitochondrial dysfunction and cell death," The American Journal of Physiology-Heart and Circulatory Physiology, vol. 302, no. 1, pp. H167-H179, 2012.

[97] R. J. Youle and D. P. Narendra, "Mechanisms of mitophagy," Nature Reviews Molecular Cell Biology, vol. 12, no. 1, pp. 9-14, 2011.

[98] R. S. Carreira, Y. Lee, M. Ghochani, Å. B. Gustafsson, and R. A. Gottlieb, "Cyclophilin D is required for mitochondrial removal by autophagy in cardiac cells," Autophagy, vol. 6, no. 4, pp. 462472, 2010.

[99] D. P. Narendra, S. M. Jin, A. Tanaka et al., "PINK1 is selectively stabilized on impaired mitochondria to activate Parkin," PLoS Biology, vol. 8, no. 1, Article ID e1000298, 2010.

[100] D. Narendra, A. Tanaka, D. F. Suen, and R. J. Youle, "Parkin is recruited selectively to impaired mitochondria and promotes their autophagy," The Journal of Cell Biology, vol. 183, no. 5, pp. 795-803, 2008.

[101] S. Geisler, K. M. Holmström, D. Skujat et al., "PINK1/Parkinmediated mitophagy is dependent on VDAC1 and p62/SQSTM1," Nature Cell Biology, vol. 12, no. 2, pp. 119131, 2010.

[102] C. Vives-Bauza, C. Zhou, Y. Huang et al., "PINK1-dependent recruitment of Parkin to mitochondria in mitophagy," Proceedings of the National Academy of Sciences of the United States of America, vol. 107, no. 1, pp. 378-383, 2010.

[103] J. Lee, Y. Nagano, J. P. Taylor, K. L. Lim, and T. Yao, “Diseasecausing mutations in Parkin impair mitochondrial ubiquitination, aggregation, and HDAC6-dependent mitophagy," Journal of Cell Biology, vol. 189, no. 4, pp. 671-679, 2010.

[104] Y. Chen, Y. Liu, and G. W. Dorn II, "Mitochondrial fusion is essential for organelle function and cardiac homeostasis," Circulation Research, vol. 109, no. 12, pp. 1327-1331, 2011.

[105] Y. Chen and G. W. Dorn II, "PINK1-phosphorylated mitofusin 2 is a parkin receptor for culling damaged mitochondria," Science, vol. 340, no. 6131, pp. 471-475, 2013.

[106] W. Ding, F. Guo, H. Ni et al., "Parkin and mitofusins reciprocally regulate mitophagy and mitochondrial spheroid formation," Journal of Biological Chemistry, vol. 287, no. 50, pp. 4237942388, 2012.

[107] T. Zhao, X. Huang, L. Han et al., "Central role of mitofusin 2 in autophagosome-lysosome fusion in cardiomyocytes," The Journal of Biological Chemistry, vol. 287, no. 28, pp. 23615-23625, 2012.

[108] D. A. Kubli and Å. B. Gustafsson, "Mitochondria and mitophagy: the yin and yang of cell death control," Circulation Research, vol. 111, no. 9, pp. 1208-1221, 2012.

[109] H. Sandoval, P. Thiagarajan, S. K. Dasgupta et al., "Essential role for Nix in autophagic maturation of erythroid cells," Nature, vol. 454, no. 7201, pp. 232-235, 2008.

[110] H. Zhang, M. Bosch-Marce, L. A. Shimoda et al., "Mitochondrial autophagy is an HIF-1-dependent adaptive metabolic response to hypoxia," The Journal of Biological Chemistry, vol. 283, no. 16, pp. 10892-10903, 2008. 
[111] Y. Lee, H.-Y. Lee, R. A. Hanna, and A. B. Gustafsson, "Mitochondrial autophagy by bnip3 involves drpl-mediated mitochondrial fission and recruitment of parkin in cardiac myocytes," The American Journal of Physiology-Heart and Circulatory Physiology, vol. 301, no. 5, pp. H1924-H1931, 2011.

[112] I. Novak, V. Kirkin, D. G. McEwan et al., "Nix is a selective autophagy receptor for mitochondrial clearance," $E M B O$ Reports, vol. 11, no. 1, pp. 45-51, 2010.

[113] J. D. Crane, M. C. Devries, A. Safdar, M. J. Hamadeh, and M. A. Tarnopolsky, "The effect of aging on human skeletal muscle mitochondrial and intramyocellular lipid ultrastructure," Journals of Gerontology A: Biological Sciences and Medical Sciences, vol. 65 , no. 2, pp. 119-128, 2010.

[114] L. M. Dillon, A. P. Rebelo, and C. T. Moraes, "The role of PGC1 coactivators in aging skeletal muscle and heart," IUBMB Life, vol. 64, no. 3, pp. 231-241, 2012.

[115] R. Cartoni, B. Léger, M. B. Hock et al., "Mitofusins 1/2 and ERR $\alpha$ expression are increased in human skeletal muscle after physical exercise," Journal of Physiology, vol. 567, no. 1, pp. 349-358, 2005.

[116] Y. S. Yoon, D. S. Yoon, I. K. Lim et al., "Formation of elongated giant mitochondria in DFO-induced cellular senescence: Involvement of enhanced fusion process through modulation of Fisl," Journal of Cellular Physiology, vol. 209, no. 2, pp. 468-480, 2006.

[117] S. Campello, F. Strappazzon, and F. Cecconi, "Mitochondrial dismissal in mammals, from protein degradation to mitophagy," Biochimica et Biophysica Acta, vol. 1837, pp. 451-460, 2014.

[118] J. Heo and J. Rutter, "Ubiquitin-dependent mitochondrial protein degradation," International Journal of Biochemistry and Cell Biology, vol. 43, no. 10, pp. 1422-1426, 2011.

[119] J. M. Heo, N. Livnat-Levanon, E. B. Taylor et al., "A stressresponsive system for mitochondrial protein degradation," Molecular Cell, vol. 40, no. 3, pp. 465-480, 2010.

[120] M. S. Willis, A. Bevilacqua, T. Pulinilkunnil, P. Kienesberger, M. Tannu, and C. Patterson, "The role of ubiquitin ligases in cardiac disease," Journal of Molecular and Cellular Cardiology, vol. 71, pp. 43-53, 2014.

[121] J. M. Predmore, P. Wang, F. Davis et al., "Ubiquitin proteasome dysfunction in human hypertrophic and dilated cardiomyopathies," Circulation, vol. 121, no. 8, pp. 997-1004, 2010.

[122] N. C. Chan, A. M. Salazar, A. H. Pham et al., "Broad activation of the ubiquitin-proteasome system by Parkin is critical for mitophagy," Human Molecular Genetics, vol. 20, no. 9, pp. 17261737, 2011.

[123] V. Azzu and M. D. Brand, "Degradation of an intramitochondrial protein by the cytosolic proteasome," Journal of Cell Science, vol. 123, no. 4, pp. 578-585, 2010.

[124] D. H. Margineantu, C. B. Emerson, D. Diaz, and D. M. Hockenbery, "Hsp90 inhibition decreases mitochondrial protein turnover," PLoS ONE, vol. 2, no. 10, Article ID e1066, 2007.

[125] A. Tanaka, M. M. Cleland, S. Xu et al., "Proteasome and p97 mediate mitophagy and degradation of mitofusins induced by Parkin," Journal of Cell Biology, vol. 191, no. 7, pp. 1367-1380, 2010.

[126] H. Wang, P. Song, L. Du et al., "Parkin ubiquitinates Drp1 for proteasome-dependent degradation: implication of dysregulated mitochondrial dynamics in Parkinson disease," Journal of Biological Chemistry, vol. 286, no. 13, pp. 11649-11658, 2011.

[127] Q. Zhang, J. Wu, R. Wu et al., "DJ-1 promotes the proteasomal degradation of Fis1: implications of DJ-1 in neuronal protection," Biochemical Journal, vol. 447, no. 2, pp. 261-269, 2012.
[128] H. Su, F. Li, M. J. Ranek, N. Wei, and X. Wang, "COP9 signalosome regulates autophagosome maturation," Circulation, vol. 124, no. 19, pp. 2117-2128, 2011.

[129] Q. Zheng, H. Su, Z. Tian, and X. Wang, "Proteasome malfunction activates macroautophagy in the heart," American Journal of Cardiovascular Disease, vol. 1, no. 3, pp. 214-226, 2011.

[130] E. J. Lesnefsky, S. Moghaddas, B. Tandler, J. Kerner, and C. L. Hoppel, "Mitochondrial dysfunction in cardiac disease: ischemia-reperfusion, aging, and heart failure," Journal of Molecular and Cellular Cardiology, vol. 33, no. 6, pp. 1065-1089, 2001.

[131] Y. Matsui, H. Takagi, X. Qu et al., "Distinct roles of autophagy in the heart during ischemia and reperfusion: roles of AMPactivated protein kinase and beclin 1 in mediating autophagy," Circulation Research, vol. 100, no. 6, pp. 914-922, 2007.

[132] P. Zhai, S. Sciarretta, J. Galeotti, M. Volpe, and J. Sadoshima, "Differential roles of gsk- $3 \beta$ during myocardial ischemia and ischemia/reperfusion," Circulation Research, vol. 109, no. 5, pp. 502-511, 2011.

[133] S. Sciarretta, P. Zhai, D. Shao et al., "Rheb is a critical regulator of autophagy during myocardial ischemia: pathophysiological implications in obesity and metabolic syndrome," Circulation, vol. 125, no. 9, pp. 1134-1146, 2012.

[134] N. Hariharan, Y. Maejima, J. Nakae, J. Paik, R. A. Depinho, and J. Sadoshima, "Deacetylation of FoxO by Sirtl plays an essential role in mediating starvation-induced autophagy in cardiac myocytes," Circulation Research, vol. 107, no. 12, pp. $1470-1482,2010$.

[135] A. Hamacher-Brady, N. R. Brady, and R. A. Gottlieb, "Enhancing macroautophagy protects against ischemia/reperfusion injury in cardiac myocytes," Journal of Biological Chemistry, vol. 281, no. 40, pp. 29776-29787, 2006.

[136] Y. Liu, S. Shoji-Kawata, R. M. Sumpter Jr. et al., "Autosis is a $\mathrm{Na}^{+}, \mathrm{K}^{+}$-ATPase-regulated form of cell death triggered by autophagy-inducing peptides, starvation, and hypoxiaischemia," Proceedings of the National Academy of Sciences of the United States of America, vol. 110, no. 51, pp. 20364-20371, 2013.

[137] C. Huang, A. M. Andres, E. P. Ratliff, G. Hernandez, P. Lee, and R. A. Gottlieb, "Preconditioning involves selective mitophagy mediated by parkin and p62/SQSTM1," PLoS ONE, vol. 6, no. 6, Article ID e20975, 2011.

[138] A. M. Andres, G. Hernandez, P. Lee et al., "Mitophagy is required for acute cardioprotection by simvastatin," Antioxidants \& Redox Signaling, 2013.

[139] Y. Maejima, S. Kyoi, P. Zhai, T. Liu, H. Li, and A. Ivessa, "Mstl inhibits autophagy by promoting the interaction between Beclin1 and Bcl-2," Nature Medicine, vol. 19, pp. 1478-1488, 2013.

[140] D. A. Kubli, X. Zhang, Y. Lee et al., "Parkin protein deficiency exacerbates cardiac injury and reduces survival following myocardial infarction," The Journal of Biological Chemistry, vol. 288, no. 2, pp. 915-926, 2013.

[141] N. Hariharan, Y. Ikeda, C. Hong et al., "Autophagy plays an essential role in mediating regression of hypertrophy during unloading of the heart," PLoS ONE, vol. 8, no. 1, Article ID e51632, 2013.

[142] F. Billia, L. Hauck, F. Konecny, V. Rao, J. Shen, and T. W. Mak, "PTEN-inducible kinase 1 (PINK1)/Park6 is indispensable for normal heart function," Proceedings of the National Academy of Sciences of the United States of America, vol. 108, no. 23, pp. 9572-9577, 2011.

[143] P. Bhandari, M. Song, Y. Chen, Y. Burelle, and G. W. Dorn II., "Mitochondrial contagion induced by Parkin deficiency in 
Drosophila hearts and its containment by suppressing mitofusin," Circulation Research, vol. 114, pp. 257-265, 2014.

[144] A. Rana, M. Rera, and D. W. Walker, "Parkin overexpression during aging reduces proteotoxicity, alters mitochondrial dynamics, and extends lifespan," Proceedings of the National Academy of Sciences of the United States of America, vol. 110, no. 21, pp. 8638-8643, 2013.

[145] A. Martin-Montalvo, E. M. Mercken, S. J. Mitchell et al., "Metformin improves healthspan and lifespan in mice," Nature Communications, vol. 4, article 3192, 2013.

[146] V. N. Anisimov, "Metformin: do we finally have an anti-aging drug?” Cell Cycle, vol. 12, pp. 3483-3489, 2013.

[147] E. Morselli, L. Galluzzi, O. Kepp et al., "Autophagy mediates pharmacological lifespan extension by spermidine and resveratrol," Aging, vol. 1, no. 12, pp. 961-970, 2009.

[148] C. He, M. C. Bassik, V. Moresi et al., "Exercise-induced BCL2regulated autophagy is required for muscle glucose homeostasis," Nature, vol. 481, no. 7382, pp. 511-515, 2012. 


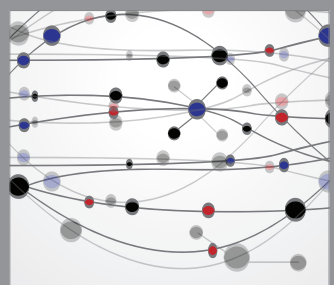

The Scientific World Journal
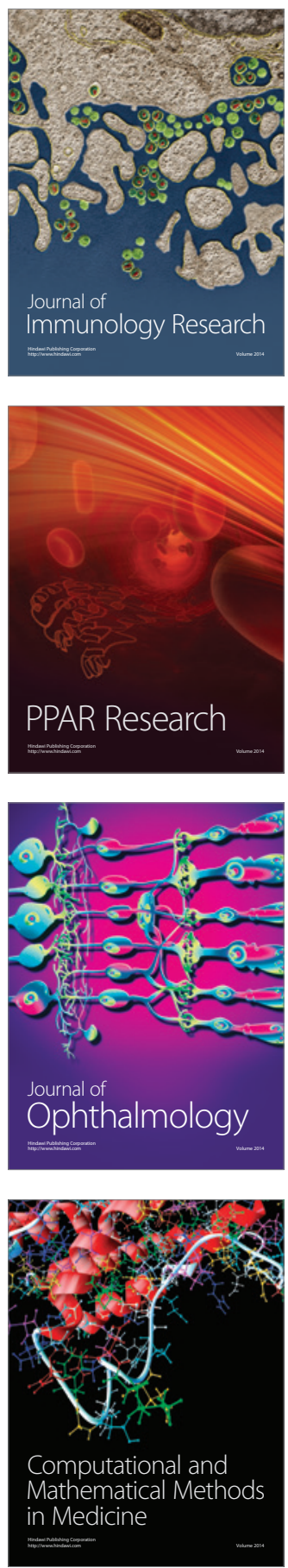

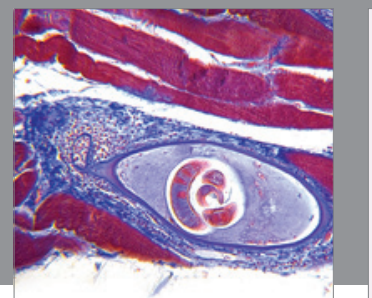

Gastroenterology

Research and Practice
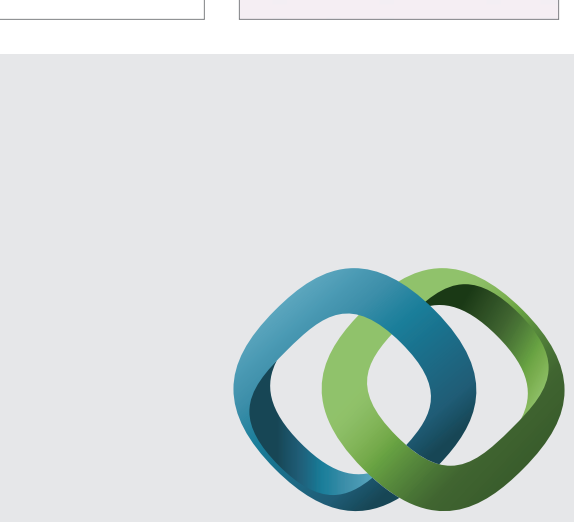

\section{Hindawi}

Submit your manuscripts at

http://www.hindawi.com
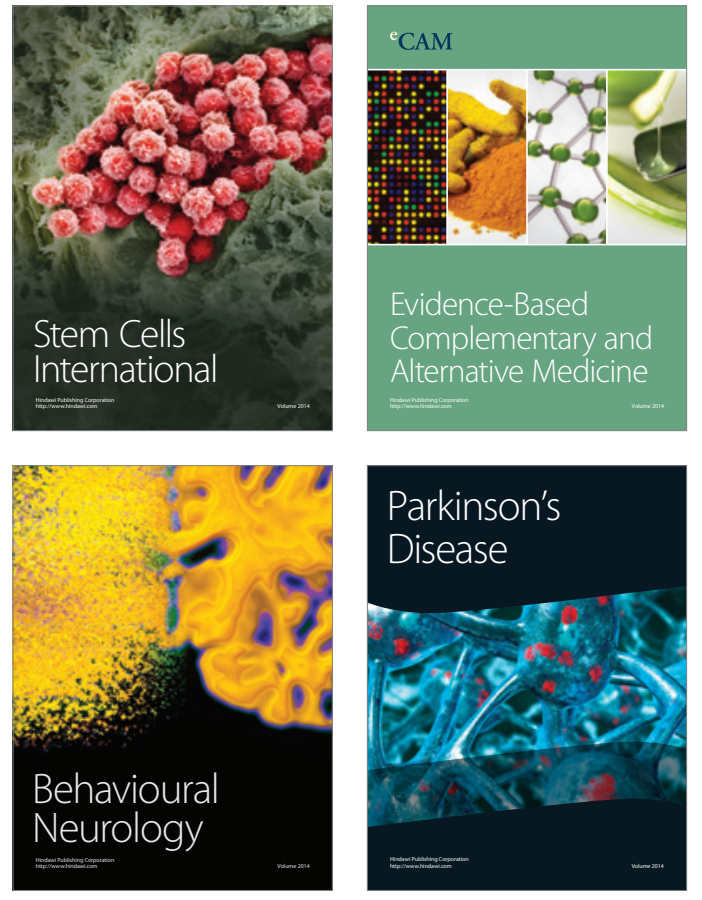
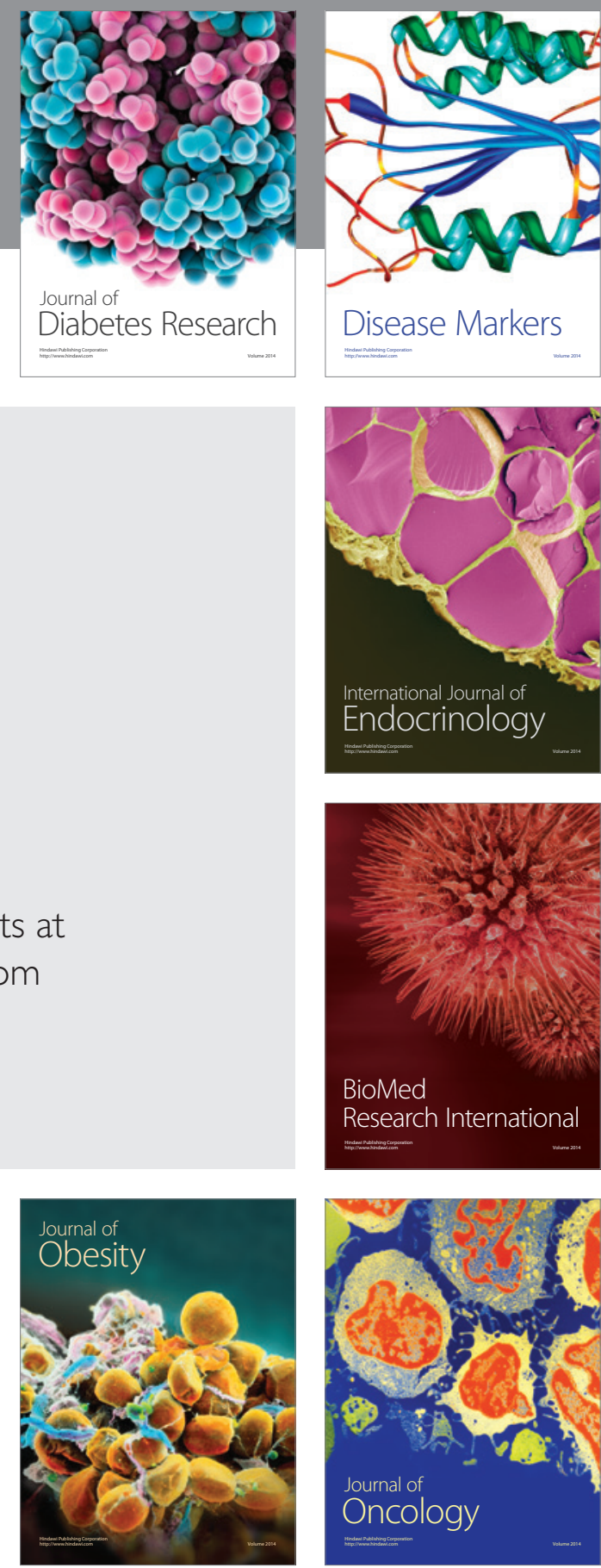

Disease Markers
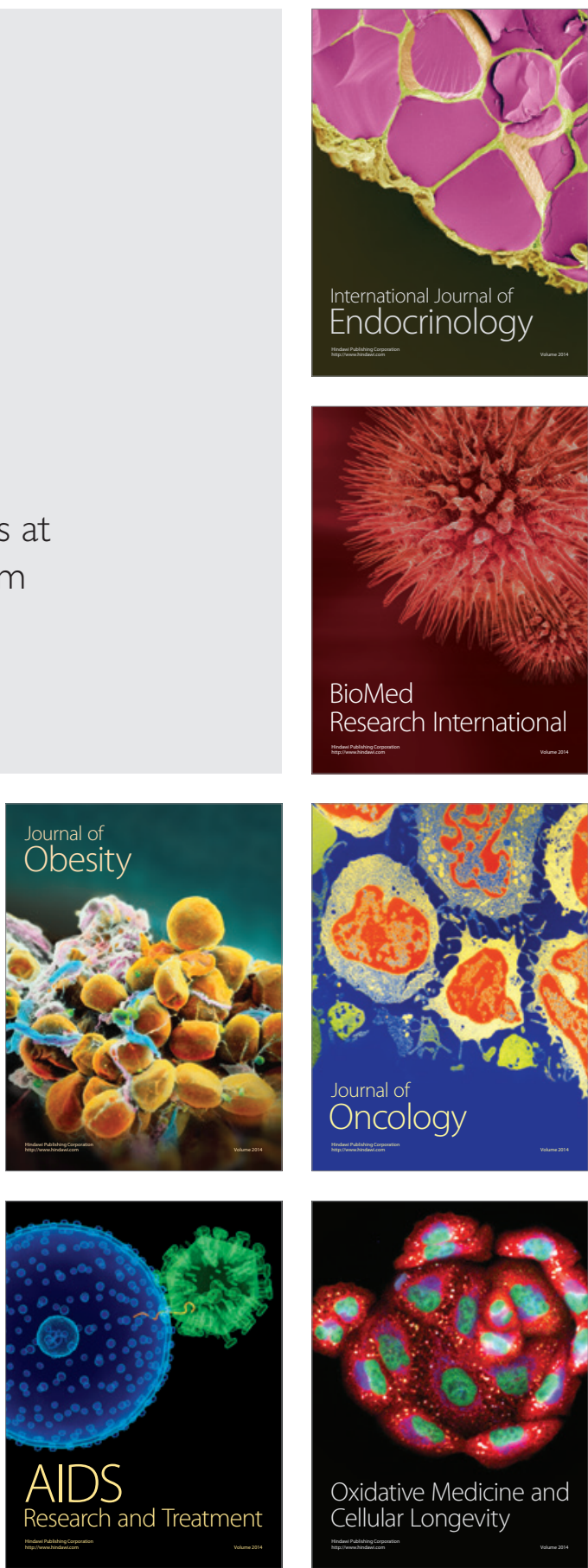